\title{
Papers
}

\section{The UK accelerated immunisation programme and sudden unexpected death in infancy: case-control study}

\author{
Peter J Fleming, Peter S Blair, Martin Ward Platt, John Tripp, Iain J Smith, Jean Golding, and the \\ CESDI SUDI research group
}

\begin{abstract}
Objectives To investigate whether the accelerated immunisation programme in the United Kingdom is associated, after adjustment for potential confounding, with the sudden infant death syndrome. Design Population based case-control study, February 1993 to March 1996. Parental interviews were conducted for each death and for four controls matched for age, locality, and time of sleep. Immunisation status was taken from records held by the parents.

Setting Five regions in England with a combined population of over 17 million.

Subjects Immunisation details were available for 93\% $(303 / 325)$ of infants whose deaths were attributed to the sudden infant death syndrome (SIDS); $90 \%$

$(65 / 72)$ of infants with explained sudden deaths; and $95 \%(1515 / 1588)$ of controls.

Results After all potential confounding factors were controlled for, immunisation uptake was strongly associated with a lower risk of SIDS (odds ratio 0.45 (95\% confidence interval 0.24 to 0.85$)$ ). This difference became non-significant (0.67 (0.31 to 1.43$)$ ) after further adjustment for other factors specific to the infant's sleeping environment. Similar proportions of SIDS deaths and reference sleeps (corresponding to the time of day during which the index baby had died) among the controls occurred within 48 hours of the last vaccination $(5 \%(7 / 149) v 5 \%(41 / 822))$ and within two weeks $(21 \%(31 / 149)$ v $27 \%(224 / 822))$.

No longer term temporal association with immunisation was found ( $\mathrm{P}=0.78)$. Of the SIDS infants who died within two weeks of vaccination, $16 \%$ $(5 / 31)$ had signs and symptoms of illness that suggested that medical contact was required, compared with $26 \%(16 / 61)$ of the non-immunised SIDS infants of similar age. The findings for the infants who died suddenly and unexpectedly but of explained causes mirrored those for SIDS infants. Conclusions Immunisation does not lead to sudden unexpected death in infancy, and the direction of the relation is towards protection rather than risk.
\end{abstract}

\section{Introduction}

The age at which infants receive their primary course of immunisation corresponds to the peak age for the inci- dence of the sudden infant death syndrome, promoting speculation that these two events might be related. During the past 20 years sporadic reports ${ }^{1-4}$ and some methodologically weak case-control studies $^{56}$ showed a possible association. However, a series of studies came to the opposite conclusion, ${ }^{7-11}$ and one raised the possibility that an accelerated immunisation programme directly contributed to a reduction in these deaths. ${ }^{12}$ All these studies share the weakness that they may be biased by residual confounding. ${ }^{13}$ In particular, an infant who is showing minor symptoms may have immunisation delayed, and infants from the most deprived and geographically mobile families are least likely to be immunised. As these factors may independently influence the risk of the sudden infant death syndrome it is essential that they feature in any analysis.

In 1990 the national immunisation programme in the United Kingdom was accelerated, with immunisation against diphtheria, tetanus and pertussis, and oral poliomyelitis given at ages 2, 3, and 4 months respectively instead of at ages 3,5 , and 9 months. Since 1992 immunisation against Haemophilus influenzae type $\mathrm{b}$ has also been given. The accelerated schedule was adopted after recognition that a common reason for low uptake of the vaccine was the mobility of young families who move out of districts before the children had completed the primary courses. ${ }^{14}$ Recent estimates suggest that $93 \%$ of infants complete the primary course by 1 year of age. ${ }^{15}$

We conducted a large case-control study of sudden unexpected death in infancy as part of the Confidential Enquiry into Stillbirths and Deaths in Infancy (CESDI SUDI study), after the changes in the immunisation programme and the reduction in the rate of the sudden infant death syndrome in the early 1990s. Findings previously reported from this study suggested that infants dying of the syndrome and those dying of explained causes share many of the same underlying factors, including high levels of deprivation, ${ }^{16}$ increased geographical mobility, ${ }^{17}$ and symptoms of illness in the 24 hours before death. ${ }^{18}$ We examine here one of the primary hypotheses of the study-a temporal relation between the accelerated immunisation programme and time of death.
Institute of Child
Health, Royal
Hospital for
Children, Bristol
BS2 8BJ
Peter J Fleming
professor of infant
health and
developmental
physiology
Peter S Blair
medical statistician
Jean Golding
professor of paediatric
and perinatal
epidemiology
Newcastle Neonatal
Service, Ward 35,
Royal Victoria
Infirmary,
Newcastle upon
Tyne NE1 4LP
Martin Ward Platt
consultant
paediatrician
Department of
Child Health,
Postgraduate
Medical School,
Royal Devon and
Exeter Hospital,
Exeter EX2 5DW
John Tripp
consultant
paediatrician
Nuffield Institute for
Health Services,
Leeds LS2 9PL
Iain J Smith
senior lecturer in
health research
Correspondence to:
P Fleming
peter.fleming@bris.
ac.uk
BMJ 2001;322:1-5 


\section{Methods}

The methods of the study are described elsewhere. ${ }^{16-20}$ Briefly, it was a large, population based, case-control study initially conducted in three former health regions (the South West, Northern, and Yorkshire regions) for two years (February 1993 to January 1995) and expanded (Wessex and Northern regions) for a third year (April 1995 to March 1996). Ethical approval was obtained in each region from the local research ethics committees. The study aimed to include all sudden unexpected deaths (both explained and unexplained) of infants aged 1 week to 1 year from a total study population of 17.7 million people. Four age matched controls for each case were selected.

An interviewer visited each control family (matched for locality) within a week of the death to collect the same data as for the index case. A period of sleep (the "reference sleep") corresponding to the time of day during which the index baby had died was identified in the 24 hours before the interviews of the control families.

Data were collected on a questionnaire by research interviewers and from medical records, including details of immunisation from records held by parents. A multidisciplinary committee established cause of death after a full paediatric postmortem examination to a standard protocol.

A modified form of the Cambridge Baby Check was included to capture signs and symptoms of illness in the final 24 hours before death or reference sleep. ${ }^{18}$ This is a check system to help to quantify illness in babies; medical contact is suggested if infants score more than 7 .

An infant was considered immunised if he or she had received any component of the programme before the last or reference sleep. A conservative estimate of the uptake of the immunisation programme was calculated as the proportion of infants who began the programme by age 90 days.

\section{Statistical methodology}

Data that were not normally distributed were described by using medians and interquartile ranges. Correlation was conducted using Pearson's coefficient. The MantelHaenszel $\chi^{2}$ test was used to test individual confounders. Odds ratios, $95 \%$ confidence intervals, and $\mathrm{P}$ values for the univariate and multivariate analyses were calculated-with the matching taken into account by using conditional logistic regression-with the SAS statistical package. ${ }^{21}$ Models were constructed with the stepwise procedure for variables significant at the $5 \%$ level in the univariate analysis.

\section{Results}

\section{Ascertainment}

Over the three years there were over 470000 births in the study area and 456 sudden unexpected deaths in infancy, of which 363 were attributed to the sudden infant death syndrome. Interviews were conducted for 325 deaths attributed to the sudden infant death syndrome (90\%), 72 of the 93 explained deaths (77\%), and the controls. Immunisation details were available for $93 \%(303 / 325)$ of the infants whose deaths were attributed to the sudden infant death syndrome, $90 \%$
$(65 / 72)$ of the infants with explained deaths, and $95 \%$ $(1515 / 1588)$ of controls.

The major causes of death among the 72 explained deaths were unrecognised infection (46\% (33)), accidental $(15 \%(11))$, congenital anomalies (14\% (10)), and non-accidental injury $(13 \%(9))$. The causes of the remaining deaths included metabolic disorders, bowel obstruction, bronchopulmonary dysplasia, and cardiomyopathy.

\section{Proportion immunised and potential confounding}

\section{Sudden infant death syndrome}

Just under half (149/303) of the SIDS infants (infants whose deaths were attributed to the sudden infant death syndrome) had begun or completed the immunisation programme, compared with two thirds $(822 / 1234)$ of the controls for the SIDS infants-a significant univariate difference (odds ratio $0.48(95 \%$ confidence interval 0.37 to 0.63$)$ ). When adjusted for matching, this significance increased $(0.23$ (0.14 to $0.37)$ ). The uptake of the programme, based on infants aged 3 months or older, was $93 \%(638 / 688)$ among the control infants and 79\% (116/146) among the index infants. The table stratifies immunisation rates for confounding factors that might explain the lower uptake among SIDS infants. The difference between the SIDS infants and the control infants was consistent across the different age groups. The proportion immunised was similar across the social classes among the controls, with reduced uptake among the index families in both the highest and lowest social strata. Moving house was associated with reduced uptake of immunisation for both groups. The uptake was slightly higher among the younger index mothers but not among the younger control mothers, and lower among larger families in both index and control families. Slightly fewer of the control infants with low birth weight or short gestation had been immunised, although this was not observed among the index infants. Increased medical contact either by admission to a special care baby unit or by subsequent hospital admission was associated with an increased uptake of immunisation for both groups; similarly, uptake was highest for the infants who had experienced a life threatening event, many of whom were seen by their doctor. Few infants had a five minute Apgar score less than 8 ; of those who did, a higher proportion of the controls were immunised.

After all of the above confounders were controlled for, immunisation uptake remained strongly associated with a lower risk of the sudden infant death syndrome (multivariate odds ratio 0.45 (0.24 to 0.85$)$ ). However, when we also controlled for highly significant risk factors in the infant's sleeping environment for the last or reference sleep-such as placing the infant prone or finding the infant with bedclothes over the head-the difference became non-significant $(0.67$ (0.31 to 1.43$))$.

We also analysed the immunisation uptake for a subgroup of infants subsequently matched for potential confounding. We selected the infants with normal birth weight $(>2500 \mathrm{~g})$ and gestational age ( $\geqslant 37$ completed weeks) who had had a normal Apgar score $(>7)$, no history of an apparent life threatening event, never been admitted to either a special care baby unit or hospital, and not moved house more than once in the past year. Preserving the age matching, we chose 
one control infant matched for social class (within one stratum), maternal age (within five years), and parity (within one child). The resultant comparison yielded 60 SIDS infants and 60 closely matched controls. In this subgroup 58\% (35) of the SIDS infants were immunised, compared with $63 \%$ (38) of the controls. At age 3 months or older, the corresponding figures were $86 \%(25 / 29)$ and $97 \%(31 / 32)$.

\section{Explained deaths}

Of the infants who died of explained causes, 54\% $(35 / 65)$ began or completed the immunisation programme, compared with $61 \%(172 / 281)$ of controls (univariate odds ratio 0.51 (0.21 to 1.26$)$ ). The uptake of the programme was $86 \%(159 / 184)$ among the control infants and 82\% (32/39) among the infants who died of explained causes. Of those who died of infection, 48\% (15/31) were immunised, compared with $58 \%(74 / 128)$ of controls $(0.44$ (0.11 to 1.65$))$.

\section{Temporal comparison}

\section{Sudden infant death syndrome}

The median age at which the first immunisation was given was 61 (interquartile range 56-71) days for SIDS infants and 59 (36-63) days for controls. The observed higher proportion of immunised controls was consistent over age (figure).

The median time from last immunisation to death was 27 (16-68) days, similar to the 29 (13-70) days until interview for controls. Five per cent $(7 / 149)$ of the immunised SIDS infants had received a vaccination in the 48 hours before death; $5 \%$ (41/822) of immunised control infants had received a vaccination in the 48 hours before the reference sleep. With the period extended to two weeks, the corresponding values were $21 \%(31 / 149)$ and 27\% (224/822). The distribution of the interval between the last vaccination and death or reference sleep did not seem to yield a particular interval in which a higher proportion of deaths occurred than expected $(\mathrm{P}=0.78)$.

\section{Explained deaths}

Of the infants whose death was explained and who had begun or completed the immunisation programme,

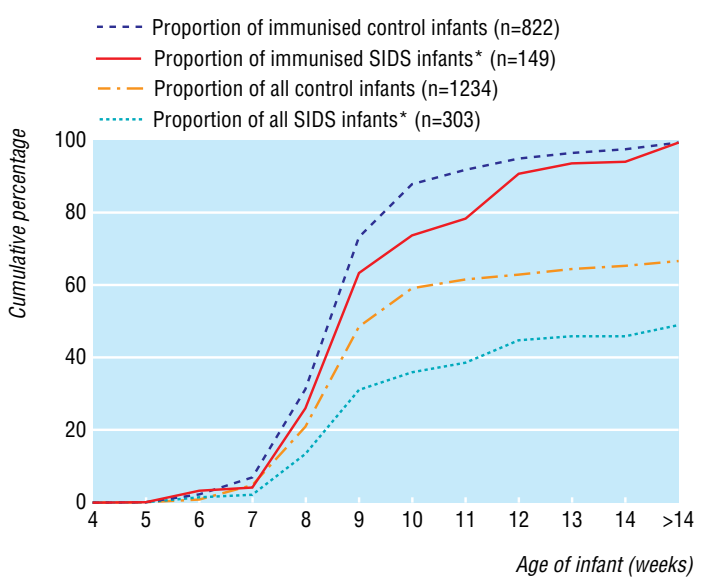

* SIDS infants = infants whose deaths were attributable to the sudden infant death syndrome Cumulative proportion of immunised infants, by age
Controlling for individual potential confounders of immunisation

\begin{tabular}{|c|c|c|c|}
\hline \multirow[b]{2}{*}{ Potential confounders } & \multicolumn{2}{|c|}{ Proportion (\%) of immunised infants } & \multirow{2}{*}{$\begin{array}{l}\text { Mantel-Haenszel pooled } \\
\text { odds ratio } \\
\text { (95\% confidence interval) }\end{array}$} \\
\hline & SIDS infants & Control infants & \\
\hline Overall & $149 / 303(49)$ & $822 / 1234(67)$ & $0.48(0.37 \text { to } 0.63)^{*}$ \\
\hline \multicolumn{4}{|l|}{ Age of infant (days)†: } \\
\hline$<91$ & $33 / 157(21)$ & 184/546 (34) & \\
\hline $91-120$ & $36 / 46(78)$ & 177/200 (89) & \\
\hline$>120$ & $80 / 100(80)$ & $461 / 488(94)$ & $0.43(0.29$ to 0.59$)$ \\
\hline \multicolumn{4}{|l|}{ Socioeconomic groupł: } \\
\hline I and II & $18 / 43(42)$ & $280 / 428(65)$ & \\
\hline III (non-manual) & $44 / 78(56)$ & 266/387 (69) & \\
\hline III (manual) and IV & $60 / 114(53)$ & $215 / 328(66)$ & \\
\hline V and unemployed & $27 / 67(40)$ & $58 / 87(67)$ & $0.50(0.38$ to 0.65$)$ \\
\hline \multicolumn{4}{|c|}{ Moved house in past year: } \\
\hline No & $82 / 159(52)$ & $651 / 977(67)$ & \\
\hline Once & $47 / 94(50)$ & 139/204 (68) & \\
\hline Twice or more & $20 / 49(41)$ & $30 / 50(60)$ & 0.51 (0.38 to 0.66$)$ \\
\hline \multicolumn{4}{|l|}{ Maternal age (years): } \\
\hline$>29$ & $29 / 62(47)$ & $271 / 410(66)$ & \\
\hline $25-29$ & $30 / 75(40)$ & $305 / 444(69)$ & \\
\hline$<25$ & $90 / 166(54)$ & $246 / 380(65)$ & 0.49 (0.37 to 0.63$)$ \\
\hline \multicolumn{4}{|l|}{ No of children in family: } \\
\hline 1 child & $47 / 84(56)$ & $354 / 529(67)$ & \\
\hline 2 or 3 children & $80 / 169(47)$ & $414 / 610(68)$ & \\
\hline$>3$ children & $22 / 50(44)$ & $54 / 95(57)$ & 0.50 (0.39 to 0.66$)$ \\
\hline \multicolumn{4}{|l|}{ Birth weight $(\mathrm{g})$ : } \\
\hline$\geqslant 2500$ & $116 / 236(49)$ & $776 / 1163(67)$ & \\
\hline$<2500$ & $33 / 67(49)$ & $39 / 64(61)$ & 0.50 (0.38 to 0.66$)$ \\
\hline \multicolumn{4}{|l|}{ Gestational age (weeks): } \\
\hline$>38$ & 49/104 (47) & 453/696 (65) & \\
\hline $37-38$ & 70/140 (50) & $319 / 461(69)$ & \\
\hline$<37$ & $30 / 58(52)$ & $41 / 66(62)$ & $0.48(0.37$ to 0.63$)$ \\
\hline \multicolumn{4}{|c|}{ Admitted to special care baby unit: } \\
\hline No & $112 / 229(49)$ & $751 / 1139(66)$ & \\
\hline Yes & $37 / 74(50)$ & $65 / 87(75)$ & $0.47(0.35$ to 0.61$)$ \\
\hline \multicolumn{4}{|l|}{ Admitted to hospital: } \\
\hline No & 97/212 (46) & $664 / 1027(65)$ & \\
\hline Yes & $51 / 88(58)$ & 157/206 (76) & 0.45 (0.35 to 0.59$)$ \\
\hline \multicolumn{4}{|l|}{ Five minute Apgar score: } \\
\hline$\geqslant 8$ & 139/277 (50) & $772 / 1170(66)$ & \\
\hline$<8$ & $6 / 15(40)$ & $20 / 25(80)$ & 0.50 (0.38 to 0.65$)$ \\
\hline \multicolumn{4}{|c|}{ Apparent life threatening event§: } \\
\hline No episodes & $128 / 262(49)$ & $792 / 1196(66)$ & \\
\hline One episode or more & 19/34 (56) & $29 / 37(78)$ & $0.48(0.36$ to 0.62$)$ \\
\hline
\end{tabular}

SIDS infants=infants whose deaths were attributed to sudden infant death syndrome.

*Unmatched odds ratio.

†Cut-offs were chosen on the basis of the infants who died within three months (some of who died after vaccination at age 2 months), those who died aged 3 to 4 months (after the second vaccination), and those who died after age 4 months and the final vaccination.

$\ddagger$ Based on highest occupational status of parent(s) and previous occupation if currently unemployed. $\S$ Any episode of lifelessness described by the parents.

$6 \%(2 / 35)$ died within 48 hours of a vaccination; $3 \%$ $(6 / 172)$ of the age matched immunised controls had been vaccinated within 48 hours of the reference sleep. With the interval extended to two weeks, the corresponding values were $17 \%(6 / 35)$ and $16 \%$ (28/172). Of the immunised infants who died of infection, $7 \%(1 / 15)$ had received a vaccination within two weeks of death, compared with $9 \%(12 / 128)$ of the immunised controls within two weeks of their reference sleep.

\section{Signs and symptoms of illness}

Sudden infant death syndrome

In the overall study $21 \%(68 / 318)$ of the SIDS infants scored $>7$ on the Baby Check (suggesting that medical advice or attention would have been required) in the 24 hours before death, compared with 7\% (97/ 
1299) of the controls in the 24 hours before the reference sleep (univariate odds ratio 4.18 (95\% confidence interval 2.73 to 6.39$)$ ). Of the SIDS infants who died within two weeks of immunisation, $16 \%(5 / 31)$ scored $>7$ on the Baby Check, compared with 26\% (16/61) of the non-immunised SIDS infants who were older than 2 months (babies under this age would rarely be immunised).

Analysing only the infants aged 2 months or older, we found no correlation, for either the index or the control group or overall, between the interval from the last immunisation to death or reference sleep and the Baby Check score measured in this latter period (Pearson's correlation coefficient $0.01, \mathrm{P}=0.70$ ).

Of the 12 signs and symptoms of illness, only two were more common among the SIDS infants (but not controls) who were immunised within two weeks of the reference sleep than among all the SIDS infants in the study. Of these 31 immunised infants, 6 were less alert than usual and 2 had blue fingernails or toenails, but these proportions were not significantly more than expected when compared with the non-immunised infants older than 2 months or with all the SIDS infants.

Among the infants who scored $>7$ on the Baby Check, 3\% (2/68) of the SIDS infants had been immunised within the previous 48 hours, compared with $5 \%$ (5/97) of the controls. The composite Baby Check score was similar in these two small groups, as were the signs and symptoms of illness.

\section{Explained deaths}

Of the infants who died of explained causes, the proportion of infants with a high Baby Check score was even more marked: $65 \%(20 / 31)$ scored $>7$, compared with $8 \%(11 / 132)$ of the age matched controls (univariate odds ratio 22.87 (5.46 to 95.83)). Only one infant died of an infection within two weeks of immunisation (within 10 days), and this infant had no signs or symptoms of illness in the 24 hours before death. Again, we found no correlation between the interval from the last immunisation to death or reference sleep and the Baby Check score measured in this period (Pearson's correlation coefficient $0.07, \mathrm{P}=0.50$ ).

\section{Discussion}

More than a third of the deaths attributed to the sudden infant death syndrome in this study occurred between the ages of 2 and 4 months, around the time that most infants in the United Kingdom were receiving all three primary immunisations against $\mathrm{Hae}$ mophilus influenza type $\mathrm{b}$, diphtheria, tetanus and pertussis, and oral poliomyelitis. For this to be more than coincidental one would expect a higher immunisation uptake among the infants who died than among age matched surviving infants, or at least some temporal pattern compatible with a reaction to immunisation. The findings from this study suggest the opposite: fewer infants whose deaths were attributed to the sudden infant death syndrome were immunised, and fewer deaths occurred than expected, both within two days and two weeks of the last vaccination, with no particular pattern beyond this time period. The findings for the infants who died suddenly and unexpectedly but of explained causes, particularly infections, mirrored those for the infants whose deaths were attributed to the sudden infant death syndrome: lower compliance, no temporal effect, and no correlation between recent immunisation and signs or symptoms of illness. Our data suggest that even when potentially confounding factors, such as family mobility, are taken into account, immunisation does not contribute to the risk of the sudden infant death syndrome and may protect against it.

Possible reactions to immunisation against diphtheria and tetanus and pertussis include fever, vomiting, and listlessness. ${ }^{7}$ Only listlessness was more common among the infants immunised within two weeks of death, and this was not significantly more than expected. In fact, among the infants whose deaths were attributed to the sudden infant death syndrome and who were older than 2 months, a greater proportion of those not immunised showed signs and symptoms of illness in the 24 hours before death that suggest medical attention would have been required.

\section{Adjustment for confounding}

Previously we have found similar characteristics among infants whose deaths were attributed to the sudden infant death syndrome and among those who died unexpectedly but of explained causes, with social deprivation and infant illness shortly before death being strong markers. ${ }^{16} 18$ A greater proportion of infants whose deaths were attributed to the sudden infant death syndrome were from socially deprived households and from younger, larger families, who tended to move home more often. These infants were also vulnerable in terms of problems at birth and further hospital admissions. However, these potential confounders did not explain, either individually or collectively, the lower than expected immunisation uptake among infants whose deaths were attributed to the sudden infant death syndrome. The uptake among the control infants reflected the national average (93\%) but the uptake among the infants who died of the syndrome was $10-15 \%$ below this. After further adjustment for other particularly strong associations related to the infants' sleeping environment, the difference in immunisation uptake was not significant but was still in the direction of immunisation being protective.

Some studies have suggested that immunisation may bring an increased risk for certain subgroups of the infant population. A case series conducted by Walker and colleagues from 1972 to 1983 in the United States suggested an increased risk of the sudden infant death syndrome within three days of primary immunisation for healthy children with normal birth weights, ${ }^{22}$ although these findings were not subsequently confirmed, ${ }^{23}$ and a study in France suggested an increased risk among infants under 3 months old. ${ }^{24}$ In our study, only a fifth of infants aged under 3 months whose deaths were attributed to the sudden infant death syndrome had begun the immunisation programme, compared with a third of the surviving infants. The subgroup analysis of these infants, closely matched for socioeconomic and clinical characteristics with surviving infants, still showed a lower uptake than expected.

\section{Implications}

One possible source of error in previous studies would be if the effect of immunisation were to increase the 


\section{What is already known on this topic}

Some studies have suggested a link between the sudden infant death syndrome and primary immunisation, but most have failed to show a link

Potential bias in the studies includes lack of a comparative control group with similar low immunisation uptake and misclassification of cause of death

\section{What this study adds}

This study investigated explained sudden infant deaths as well as the sudden infant death syndrome and took into account potential bias

After confounding was controlled for, immunisation uptake was lowest among the infants who died, with no temporal relation or correlation with signs and symptoms of illness

risk of sudden unexpected deaths that were subsequently attributed to infection or other specific causes and thus not registered as the sudden infant death syndrome. We avoided this bias by including all infants who died suddenly of identified causes.

Shann has suggested, on the basis of observations from trials in developing countries, that measles vaccination may confer protection from death due to causes other than measles itself. ${ }^{25}$ Our data are consistent with the hypothesis that the standard primary course of immunisation may also have a non-specific protective effect on the risk of death in infancy, or alternatively that failure to begin the course may be a marker of family organisation where the sudden infant death syndrome may be more frequent.

We therefore conclude that the accelerated immunisation programme in the United Kingdom is not associated with sudden unexpected death in infancy, whether the death is explained or unexplained. These data re-emphasise the importance of rigorous analysis of temporal associations of apparent significance.

Contributors: PJF, PSB, MWP, JT, IJS, and JG were all involved in the concepts and design from the beginning of the study. The analysis was conducted by PSB under the supervision of the others, and all authors were involved in the interpretation of the data, drafting, and revisions. PJF, PSB, JT, and IJS supervised data collection for the whole study period within their particular regions, MWP for the final year of the study.

Funding: The study was supported by research grants from the National Advisory Body for Confidential Enquiry into Still- births and Deaths in Infancy, the Foundation for the Study of Infant Deaths, and the Babes in Arms charity.

Competing interests: None declared.

1 Hutcheson R. DTP immunization and sudden infant death-Tennessee. Morb Mortal Wkly Rep 1979;28:131-2.

2 Solberg LK. DTP vaccination, visit to child care health centre and sudden infant death syndrome (SIDS); evaluation of DPT vaccination. Oslo: Health Council, 1985:1-19.

3 Roberts SC. Vaccination and cot deaths in perspective. Arch Dis Child 1987;62:754-9.

4 Flahault A, Messiah A, Jougla E, Bouvet E, Perin J, Hatton F. Sudden infant death syndrome and diphtheria/tetanus toxoid/pertussis/ poliomyelitis immunisation [letter]. Lancet 1988;1:582-3.

5 Torch WA. Diphtheria-pertussis-tetanus (DTP) immunization; a potential cause of sudden infant death syndrome (SIDS) [abstract]. Neurology 1982;32:A169.

6 Baraff LJ, Ablon WJ, Weiss RC. Possible temporal association between diphtheria-tetanus toxoid-pertussis vaccination and sudden infant death syndrome. Pediatr Infect Dis 1983;2:7-11.

7 Hoffman HJ, Hunter JC, Damus K, Pakter J, Peterson D, van Belle G, et al. Diphtheria-tetanus-pertussis immunization and sudden infant death: results of the National Institute of Child Health and Human Development Co-operative epidemiological study of sudden infant death Development Co-operative epidemiological study of
syndrome risk factors. Pediatrics 1987;79:598-611.

8 Taylor EM, Emery JL. Immunisation and cot deaths [letter]. Lancet 1982;ii:721.

9 Cameron MH, Williams AL. Development and testing of scoring systems for predicting infants with high-risk of sudden infant death syndrome in Melbourne. Aust Paediatr J 1986;22(suppl 1):37-45.

10 McGlashan ND. Sudden infant deaths in Tasmania, 1980-1986: A seven year prospective study. Soc Sci Med 1989;29:1015-26.

11 Mitchell EA, Stewart AW, Clements M, Ford RPK. Immunisation and the sudden infant deaths syndrome. Arch Dis Child 1995;73:498-501.

12 Dwyer T, Ponsonby A-L, Blizzard L, Newman N, Cochrane JA. The contribution of changes in the prevalence of prone sleeping position to the decline in sudden infant death syndrome in Tasmania. JAMA 1995;273:783-9

13 Fine PE, Chen RT. Confounding in studies of adverse reactions to vaccines. Am J Epidemiol 1992;136:121-35.

14 Jeffries S, McShane S, Oerton J, Victor SR, Beardow R. Low immunization uptake rates in an inner-city health district: fact or fiction? J Public Health 1991;13:312-7.

15 Platt MJ. Child health statistics review. Arch Dis Child 1998;79:523-7.

16 Leach CEA, Blair PS, Fleming PJ, Smith IJ, Ward Platt M, Berry PJ, et al. Sudden unexpected deaths in infancy: similarities and differences in the epidemiology of SIDS and explained deaths [abstract]. Pediatrics 1999;104:953. (Entire article available at www.pediatrics.org/cgi/ content/full/104/4/e43)

17 Fleming PJ, Blair PS, Bacon C, Berry J, eds. Sudden unexpected death in infancy. The CESDI SUDI studies. London: Stationery Office, 2000.

18 Ward Platt M, Blair PS, Fleming PJ, Smith IJ, Cole TJ, Leach CEA, et al. A clinical comparison of SIDS and explained sudden infant deaths. How healthy and how normal? Arch Dis Child 2000;82.98-106.

19 Blair PS, Fleming PJ, Smith IJ, Ward Platt M, Young J, Nadin P, et al. Babies sleeping with parents: case-control study of factors influencing the risk of sudden infant death syndrome. BMJ 1999;319:1457-62.

20 Blair PS, Nadin P, Cole TJ, Fleming PJ, Smith IJ, Ward Platt M, et al. Weigh gain and sudden infant death syndrome. Changes in weight z-scores may identify infants at increased risk. Arch Dis Child 2000;82:462-8.

21 SAS Institute. SAS technical report P-229, SAS/STAT software: changes and enhancements. Release 6.07. Cary, NC: SAS Institute, 1992.

22 Walker AM, Jick H, Perera DR, Thompson RS, Knauss TA Diphtheria-tetanus-pertussis immunisation and sudden infant death syndrome. Am J Public Health 1987;77:945-51.

23 Griffin MR, Ray WA, Livengood JR, Schaffner W. Risk of sudden infant death syndrome after immunisation with the diphtheria-tetanuspertussis vaccine. N Engl J Med 1988;319:618-23.

24 Jonville-Bera AP, Autret E, Laugier J. Sudden infant death syndrome and diphtheria-tetanus-pertussis-poliomyelitis vaccination status. Fundam Clin Pharmacol 1995;9:263-70.

25 Shann S. A little bit of measles does you good. BMJ 1999;319:4-5

(Accepted 16 January 2001) 